\section{Making smarter immunotherapies}

\section{Mohammad A.A. Ibrahim and Frances M.Gotch}

Imperial College School of M edicine

Department of Immunology

Chelsea \& Westminster Hospital

369 Fulham Road

London SW 10 9N H, UK.

The design of effective prophylactic and therapeutic immunotherapy remains the "Holy Grail" of research into the immunological responses to infectious diseases. So, how should we go about manipulating the immune system successfully against microbes? The traditional approach has been to rely on "evolutionary wisdom". Ancient pathogens (such as smallpox) have exerted sustained evolutionary pressure on the vertebrate immune systems to develop protective immunity. These kinds of microbes have been used successfully as attenuated or killed vaccines. However, many microbes including HIV do not retain their immunogenicity when killed or attenuated. Perhaps for the more recently evolved pathogens, to avoid unnecessary human suffering and loss of life, we must rely on OUR wisdom, rather than mimic successful examples of protective immunity to infection.

One consideration should be the paradigm shift in our understanding of the prerequisites of immunity. The original twosignal model of lymphocyte activation no longer appears sufficient to explain initiation of immunity. Newer models emphasize the role of microbial ${ }^{1}$ and non-microbial pathways $^{2,3}$. These models have brought dendritic cells (DCs) to center stage as promising targets for intervention for immunotherapy and vaccine development, and shift the emphasis from the "antigen or epitope" towards the "adjuvant". Important advances in the area of microbial signaling have been made, such as the discovery of toll-like receptors (TLRs) and their role in immunity to microorganisms ${ }^{4}$. However, most of these so-called pattern recognition receptors (PRRs) including the TLRs are more likely to participate in immunity to ancient pathogens, because by definitionand from an evolutionary perspective- it is only possible to encode PRRs in the germline if there is persistent selective pressure.

The discovery that DCs can be activated by cell death ${ }^{5}$, without the need for a microbial signal, certainly opens the way to a new era of rational vaccine design. We should no longer need to use the microbial adjuvant effect given the opportunity of an "endogenous adjuvant". It is likely that the immune response to noxiousness is so deeply rooted in evolution that it could be the most robust adjuvant pathway. Identification of the signals involved in the activation of immune cells by tissue injury may reveal both the soluble and cell surface structures involved. Combining these with candidate pathogen antigens could provide the basis of effective, safe and practical vaccines. This would be preferable to dendritic cell immunotherapy because the latter, although effective, may not be suited for large-scale vaccine efforts, especially in developing countries.

So, how do we see the future of immunotherapy to infections? Three areas are important. First, antigen-specific immunopotentiation. This would include DNA vaccines, combinations of antigen and cytokines, and antigen combined with danger/injury signals. The second area is antigen-specific immunomodulation, possibly useful for infections associated with immunopathology, such as respiratory syncytial virus. Third, is non-specific immunopotentiation. Advances here would enable us to promote immune reconstitution following infections that destroy the immune system.

Prophylactic and therapeutic immunotherapy is badly needed for major human pathogens responsible for large-scale morbidity and mortality. The time has come for a focused effort to create rational, safe and practical immunotherapies suitable for global applications.

1. Janeway, C.A. Jr The immune system evolved to discriminate infectious nonself from noninfectious self. Immunol. Today $\mathbf{1 3}$ 11-16 (1992).

2. Matzinger, P.Tolerance, danger, and the extended family. Annu. Rev. Immunol. 12, 991-1045 (1994).

3. Ibrahim, M.A., C hain, B.M. \& Katz, D.R. The injured cell: the role of the dendritic cell system as a sentinel receptor pathway. Immunol.Today 16, 181-186 (1995).

4. Brightbill, H.D. et al. Host defense mechanisms triggered by microbial lipoproteins through toll-like receptors. Science $\mathbf{2 8 5}$ 732-736 (1999).

5. Gallucci, S., Lolkema, M. \& Matzinger, P. N atural adjuvants: endogenous activators of dendritic cells. Nature Med. $\mathbf{5}$, 1249-1255 (1999). 